\title{
Knowledge management and innovation: a bibliometric case study as technology transfer about pectin
}

\author{
Santos Pedraza Guevara ${ }^{1}$, João Luiz Kovaleski ${ }^{2}$, Maria Helene Giovanetti Canteri ${ }^{3}$, Maria Elena Ibañez \\ Ezequiel ${ }^{4}$, Revenli Fernanda Do Nascimento ${ }^{5}$
}

${ }^{1}$ Biochemistry Laboratory, Postgraduate Program in Production Engineering, Ponta Grossa Federal University of Technology - Paraná (UTFPR), PR, câmpus Ponta Grossa, Av. Monteiro Lobato, s/n - Km 04, Ponta Grossa, PR CEP 84016-210, Brazil

2 Department of Post-graduation in Production Engineering, Federal University of Technology - Parana (UTFPR) câmpus Ponta Grossa, Av. Monteiro Lobato, s/n - Km 04, Ponta Grossa, PR CEP 84016-210, Brazil

${ }^{3}$ Biochemistry Laboratory, Food Department, Ponta Grossa Federal University of Technology - Paraná (UTFPR) câmpus Ponta Grossa, Av. Monteiro Lobato, s/n - Km 04, Ponta Grossa, PR CEP 84016-210, Brazil

${ }^{4}$ Foodomics Laboratory, Bioactivity and Food Analysis Department, Institute of Food Science Research (CIALCSIC), Nicolas Cabrera 9, Campus UAM Cantoblanco, 28049 Madrid - SPAIN

${ }^{5}$ Biochemistry Laboratory, Postgraduate Program in Production Engineering, Ponta Grossa Federal University of Technology - Paraná (UTFPR), PR, câmpus Ponta Grossa, Av. Monteiro Lobato, s/n - Km 04, Ponta Grossa, PR CEP 84016-210, Brazil

Recibido el 1 de junio del 2018. Aceptado el 13 de junio del 2018

DOI: https://doi.org/10.33017/RevECIPeru2018.0001/

\section{Resumen}

Los medios de información online hoy en día son muy amplios, y la metodología de búsqueda si no se tiene el conocimiento previo en cualquier área del conocimiento hace que leamos información inútil. Este artículo demuestra la manera de cómo realizar búsquedas basándose en un estudio de caso en pectinas, utilizando Methodiordinatio como transferencia de tecnología. Realizando un análisis bibliométrico basado en palabras claves "Anthropotechnology, Knowledge management and innovation, technology transfer, pectin" en tres bases de datos, Scopus, ScienceDirect y Web on Science. La transferencia de conocimiento y fácil comunicación al lector, debe tener la capacidad de desarrollar búsquedas de información no ambigua ni compleja. Por lo que se puede esperar que proporcionen a los investigadores herramientas con ventajas que no se pueda imitar ni competir fácilmente, estimular la investigación, despertando principal interés, con especial referencia en innovación en pectinas. La revisión se refiere a la bibliometría con respecto al estudio de caso de pectinas en el área de ingeniería III y algunas otras áreas importantes de investigación. Se seleccionaron primero $32 \%$ en área de interés; el $40 \%$ de las otras áreas sólo se tomó un 3\%. Los países que más investigaciones realizaron fueron la India y Spain, seguido por Brazil, China, Italia, Belgica, Malaysa, France, Turkey e Iran. Las mayores publicaciones fueron en la revista International Journal of Food Science \& Technology. De las otras áreas el mayor número de artículos publicados en ciencias agrarias, seguido de biotecnología y ciencia de los alimentos. Se utilizaron referenciadores bibliográficos como Zotero, EndNote, y JabRef, ayudando a organizar los artículos de manera simple. Y por último están cubiertas en esta revisión, la situación actual sobre fuentes de extracción de pectinas, tal que en los resultados se encontraron la mayor cantidad de materias primas, los residuos agroalimentarios; mencionando la recuperación, sugerencias de valorización de los cultivos, utilizando nuevas tecnologías, nuevas tendencias, desafíos, incluida la reducción de pérdidas posteriores para mejorar la seguridad alimentaria, así como mantener la sostenibilidad, prácticas agrícolas y un impacto ambiental mínimo. 
Descriptores: Antropotecnología, Gestión del conocimiento e innovación, transferencia de tecnología, pectina Abstract

The online information media nowadays are very broad and if the research methodology does not have prior knowledge in any area, the information may be useless. This article demonstrates a way of searching based on a case study in pectins, using Methodiordinatio as technology transfer, by mean of the bibliometric analysis based on keywords "Anthropotechnology, Knowledge management and innovation, technology to transfer, pectin" in data bases, Scopus, ScienceDirect and Web on Science. The transfer of knowledge and easy communication to the reader must have the capacity to develop searches of non neither ambiguous nor complex information, providing to the researches tools with advantages that cannot be imitated or easily competed, stimulate research, arousing major interest, with special reference to pectins in innovation. The revision about the bibliometric with respect to the case study of pectins, in the area of engineering III and some other important areas of investigation. First $32 \%$ were selected in area of interest, of the $40 \%$ of the other areas only a volume of $3 \%$. The countries with more investigations were India and Spain, followed by Brazil, China, Italy, Belgium, Malaysia, France, Turkey and Iran. The greater amount of publications was in the magazine International Journal of Food Science \& Technology. In the other areas the greater number of articles was published in Agrarian Sciences, followed of Biotechnology and Food Science. Bibliographical tools such as Zotero, EndNote, and JabRef were used, helping to organize articles of simple way. Finally, the present situation on sources of pectins extraction, the agro alimentary wastes as raw material, mentioning the recovery, suggestions the valuation, using new technologies, new tendencies, challenges, including the reduction of later losses to improve the food security, as well as to maintain the sustainability practical agricultural and a minimum environmental impact were exposed.

\section{Keywords: Anthropotechnology, Knowledge management and innovation, technology transfer, pectin}

\section{Introduction}

The process of knowledge transfer is dynamic and continuous and includes a learning process for assimilation, and a series of activities that lead from the adoption to the acceptance. The communication of knowledge once acquired, can be written or verbal. The scientific community should be aware of the possible barriers to the dissemination of information if it has the intends to promote the transfer of knowledge [1].

In practice, is improves the competence and the quality of the information. It is why the acquired knowledge must be applied so that they are conserved; in such a way that it allows the scientific community to learn, more than the own knowledge.

The center in knowledge has led to a greater attention towards information technology [2 3]. Identify knowledge is based on great advantage, since it can develop, retain and transfer knowledge [4]. In many studies there are positive connections between $R$ \& $D$ efforts (research plus development) to generate new ideas and innovation.
The exchange of information throughout the research process provides basic for the accumulation of knowledge production and scientific progress [5]. In this context, the amount of information found on Web pages is numerous, so the number of scientific publications in various Journals has increased considerably in recent years.

Two factors that contribute to this have been observed, firstly, new technologies that provide research with new scientific research, which supports the emergence of new studies; second, the need for specialization and construction of new knowledge, imposed by markets and knowledge society, which lead to the search and dissemination of new knowledge. This he is only one of several different metric possible to be used to offer investigation activity, justifying why the university is considered the best place to precede investigation initiatives and to make knowledge transfer.

The main result of the scientific activities can be found in a series of periodic Journals and the scientific publications play an important role as primary raw material, constructing scientific knowledge to accelerate the processes of the technological innovation. Often the scientists create new scientific tools search of information [6]. 
In order to detect new dominions of investigation it is to analyze the amount of scientific publication citations [6, 7].

According to de Solla Price (1965) the scientists have tendency to mention more recent articles; and they may be excluding common articles that they deserve inclusion.

In this sense, this article is organized of the following way: revision of literature, research methodology, finally, discussion and conclusion that summarize the results, analyzes its implications of the theory and practice of the case study, and provides possible suggestions for future research. For the case study, the compilation data were carried out using Scopus, ScienceDirect and Web of science databases [8]. The study of selected case was the pectins, for with methodiordinatio [5].

\section{Theoretical Background}

\subsection{Anthropotechnology}

The anthropotechnology is developed from diverse studies of technology transfer, from the technical and organizational point of view.

Anthropotechnology is based on a discipline to solve problems, placed by the technology transfer, which establish the diverse theoretical bases of a new knowledge [9].

For a long time, the logic of industrial development used by international development agencies, induced developing countries in an intensive way such as: equipment, machines, factories, scientific knowledge, originating the term transfer of technology.

Through the ages, the human being has sought methods and work processes that reduce effort, to have good results.

The technology can be considered as a powerful force in the sense of being able to extend the mental capacities, with the computer revolution $[9,10]$.

The transfer of knowledge has been considered a relatively important work as part of knowledge management [11], different types from knowledge require diverse ways of transfer [12], such as: who is the objective receiver, type, nature of the operation of knowledge to transfer in agreement proposed by Dixon cited for [12, 13].

The transfer of technologies [14] is carried out in various types and areas of knowledge and assumes different types of modalities executed by diverse reasons and it extends several academic disciplines and professions [13].

Nowadays, postgraduate programs provide a diverse knowledge transfer; as well as different methodologies for the collection of research data, the use of the keywords in any search tool Webbased search tool, quickly gives credibility, the key to writing an article.

Although there is a professional society dedicated to the TT (Journal of Technology Transfer), where there are scientific articles on TT related to companies, but the studies of research methodologies in databases related to technology transfer are few and none hydrocolloids (pectins); so this article promises to serve as a basis as an organizational framework and / or summarize the data related to pectins and the relationship with anthropotechnology

\section{Research methodology}

\subsection{Sample and data collection}

This study uses Methodiordinatio, a methodology that proposes to select and to classify the scientific documents, including the impact factor, the number of citations and the year of publication.

\subsection{Study of case}

The case study represents a qualitative investigation.

A case analysis is a good departure point in the inductive process of the theory Yin, 1988 quoted by Tseng, 2008, since it allows the researcher to observe and collect information about new and unknown natural phenomena that had never been studied before [15].

The intent of this case study is to gather information from the article using keywords: Anthropotechnology, Knowledge management and innovation, technology transfer, pectin; the information can be visualized in the tables and images with the main descriptions in pectin such as: 
raw material, extraction method, parameters and yield.

\subsection{Selection of the case}

The Pectin

This substance is commercially obtained from apple pomace, grapefruit and citrus peel [16]. Most commercial pectin is obtained from lemon peel, lime, passion fruit and apple pomace [2, 14, 17].

In the conventional way, the pectin is extracted in acid solution to temperatures around $80^{\circ} \mathrm{C}$ by approx. 1 hour with continuous agitation [18]. In the industry, the pectin is extracted in acid conditions with high temperature [19].

The acid residual waters are a great environmental limitation, for today there are studies about other extraction methods that are respectful with the environment, such as of ultrasound [18, 20, 21], microwaves $[18,22]$ and subcritical water [20].

The properties and functionality of the pectin depend to a great extent on the structural characteristics of these polysaccharides, determined by the source, as well as treatment and conditions of extraction [23, 24].

This study of case allows to finding articles with different methodologies used for pectin extraction, its yield, raw material of extraction; using the method Methodiordinatio, so that tacit and explicit knowledge takes place guided by the practice in pectins.

In general, the researchers find direct interferences in the time of information searches to write a thesis project or an article, resulting in poorly performed work.

Using this research method, the knowledge produced can serve as a reality to concur to an action, and that the researchers in hydrocolloid subjects (pectins) can compare data; on the other hand, it is based on the methodology developed by [5] "Methodiordinatio", it is promising since it allows the systematic selection and classification of scientific articles using mathematical formulas. The steps information search steps in the High Impact Journal are shown in the table 1.
Table 1: Phases search

and selection of information

\begin{tabular}{ll}
\hline Phases & Description \\
\hline$(1)$ & Determination of key words \\
$(2)$ & Search in the main data bases: Scopus, \\
& CienceDirect, Web of Science \\
(3) & Filter or bibliometrics in data bases \\
(4) & Article extraction using three referrers: \\
& EndNote (version: X8), Zotero \\
& (version:4.0.26) and JabRef \\
& (version:2.10) according to \\
(5) & Mrticle analysis \\
& Methodiordinatio \\
(6) & Decision of article selection
\end{tabular}

Source: the authors

3.4. Study of case - Articles of extraction of pectin. Table 2 , table 3 and table 4 , shows collected data, as pectin extraction parameters.

\section{Result}

Using the Methodiordinatio, in the search of information we found 155 articles and we used 74 papers in all the revision. For the study of case, 34 papers were selected.

According the results, $32 \%$ of all the researched in Journals belong to the area of Engineering III, but only 19 Journals were selected. $40 \%$ of articles belong to the area of Food Science and $3 \%$ were selected single, $4 \%$ belong to Agrarian Sciences and it was selected $2 \%$ equal to Biotechnology; $1 \%$ belongs to Environmental Sciences as showed in Figure 1.

About the Figure 2, in relation to countries, we find 10 countries, distributing $21 \%$ India, $21 \%$ Spain, Brazil with $16 \%$, the smaller percentage by China and Italy. Belgium, Malaysia, France, Turkey and Iran, with $5 \%$ reach one.

All these researches were published in Journals of high impact is to say $A 1, A 2, B 1$ and $B 2$ respectively (Figure 3). 
Table 2: Summary of results of bibliographical review. Number of Ranking according to Methodiordinatio. Raw material of pectin obtain, method of extraction in specialized Journals from Engineering III (1-20) and group of other areas of research

\begin{tabular}{|c|c|c|c|c|c|c|c|}
\hline $\begin{array}{l}\text { Ranking } \\
\text { number }\end{array}$ & Fruit & Method of extraction & Conditions & $\mathrm{pH}$ & Temperature & $\begin{array}{l}\text { Extraction } \\
\text { time }\end{array}$ & $\begin{array}{c}\text { Yield } \\
\text { (Galacturonic } \\
\text { Acid (GalA)) } \\
\end{array}$ \\
\hline 1 & Durian rind (Durio zibethinus) & $\begin{array}{l}\text { Solid-liquid [25] ratio (1: 5-1: } 15 \\
\mathrm{~g} / \mathrm{ml})\end{array}$ & $\begin{array}{l}\text { SL ratio 1:10 } \\
\mathrm{g} / \mathrm{ml}\end{array}$ & 2.8 & $86^{\circ} \mathrm{C}$ & $43 \mathrm{~min}$ & $(9.1 \% \pm 0.5 \%)$ \\
\hline 2 & $\begin{array}{l}\text { Thorny orange (Poncirus trifoliata) } \\
\text { sweet orange (Citrus sinensis) }\end{array}$ & Electromagnetic induction & - & 1.2 & $80^{\circ} \mathrm{C}$ & $90 \mathrm{~min}$ & $24 \%(w / w)$ \\
\hline \multirow[t]{2}{*}{3} & \multirow[t]{2}{*}{ Grapefruit peel (Citrus×paradisi) } & $\begin{array}{l}\text { Ultrasound-assisted heating } \\
\text { extraction (UAHE) } \\
\text { Power intensity } 12.56 \mathrm{~W} / \mathrm{cm}^{2}\end{array}$ & \multicolumn{2}{|c|}{$\begin{array}{l}\text { Furthermore, UAHE pectin } \\
\text { possessed lower viscosity, } \\
\text { molecular weight and } \\
\text { degree of etherification, but } \\
\text { higher degree of branching } \\
\text { and purity than CHE pectin }\end{array}$} & $66.71^{\circ} \mathrm{C}$ & $27.95 \mathrm{~min}$ & $27.34 \%$ \\
\hline & & $\begin{array}{l}\text { Conventional heating extraction } \\
\text { (CHE) }\end{array}$ & - & - & $80.1^{\circ} \mathrm{C}$ & $73.9 \mathrm{~min}$ & $11.12 \%$ \\
\hline 4 & $\begin{array}{l}\text { Passion fruit peel (Passiflora } \\
\text { edulis f. flavicarpa) }\end{array}$ & \multicolumn{2}{|c|}{$\begin{array}{l}\text { Microwave-power ( } 628 \mathrm{~W}) \text {. Tartaric acid emerged } \\
\text { as the best extracting agent in terms of yield } \\
(18.2 \%) \text {, however, the obtained pectin exhibited } \\
\text { low purity and low degree of etherification. The } \\
\text { time and microwave-power significantly affects } \\
\text { the yield of pectin extraction with both nitric and } \\
\text { tartaric acids. }\end{array}$} & \multicolumn{2}{|c|}{$\begin{array}{l}\text { Degree of etherification } \\
\text { (64.56\% for acetic acid and } \\
64.15 \% \text { for nitric acid) }\end{array}$} & $9 \min$ & 13 and $12.9 \%$ \\
\hline 5 & $\begin{array}{c}\text { Pasion fruit peel } \\
\text { (Passiflora edulis) }\end{array}$ & $\begin{array}{l}\text { Conventional heating using HPP } \\
\text { (High pressure assisted) as } \\
\text { pretreatment. Solid/liquid ratio } \\
1: 30\end{array}$ & $300 \mathrm{MPa}$ & - & $50^{\circ} \mathrm{C}$ & $20 \mathrm{~min}$ & $\begin{array}{r}\text { At } 100^{\circ} \mathrm{C} 17 \\
\text { min is obtained } \\
14.34 \%\end{array}$ \\
\hline 6 & Orange peel (Citrus sinensis) & $\begin{array}{l}\text { Microwave assisted extraction. } \\
\text { solid-liquid ratio of } 1: 16.9 \mathrm{~g} / \mathrm{ml}\end{array}$ & $\begin{array}{l}\text { Microwave } \\
\text { power of } 422 \\
\text { W }\end{array}$ & 1.4 & - & $\begin{array}{l}\text { Irradiation } \\
\text { time } 169 \mathrm{~s}\end{array}$ & $19.24 \%$ \\
\hline
\end{tabular}




\begin{tabular}{|c|c|c|c|c|c|c|c|}
\hline 7 & $\begin{array}{l}\text { Carcia papaya L. peel (Papaya } \\
\text { carica L.) }\end{array}$ & $\begin{array}{l}\text { Solid-liquid ratio of } 1: 15 \mathrm{~g} / \mathrm{ml} . \\
\text { Microwave assisted extraction }\end{array}$ & $\begin{array}{c}\text { Microwave } \\
\text { power of } 512 \\
\text { w }\end{array}$ & 1.8 & - & $140 \mathrm{~s}$ & $25.41 \%$ \\
\hline $\begin{array}{l}\text { Ranking } \\
\text { number }\end{array}$ & Fruit & Method of extraction & Conditions & $\mathrm{pH}$ & Temperature & $\begin{array}{l}\text { Extraction } \\
\text { time }\end{array}$ & $\begin{array}{c}\text { Yield } \\
\text { (Galacturonic } \\
\text { Acid (GalA)) }\end{array}$ \\
\hline 8 & Apple Pomace (Malus domestica) & \multicolumn{2}{|c|}{$\begin{array}{l}\text { Ethanol used in pectin precipitation at } \mathrm{pH} 1.5 \\
\text { increases the yield of the precipitate }\end{array}$} & 1.5 to 2 & 80 to $90 \circ \mathrm{C}$ & 1 to $3 \mathrm{~h}$ & $3.4 \%$ to $8.9 \%$. \\
\hline 9 & $\begin{array}{l}\text { 'Pera' sweet orange (Citrus } \\
\text { sinensis } L \text { L. Osbeck.) }\end{array}$ & 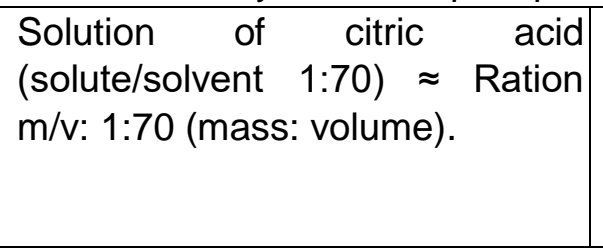 & $650 \mathrm{rpm}$ & 2.5 & $80^{\circ} \mathrm{C}$ & $120 \mathrm{~min}$ & $\begin{array}{r}11.26 \% \\
\text { (albedo) } \\
8.51 \% \\
\text { (flavedo) } \mathrm{g} \\
\text { pectin/g dry) }\end{array}$ \\
\hline 10 & $\begin{array}{l}\text { Orange pomace (Citrus sinensis } \\
\text { L.) }\end{array}$ & \multicolumn{2}{|c|}{$\begin{array}{l}\text { The yield of extraction with citric acid was } 17.75 \% \\
\text { and amid condition of extraction with nitric acid } \\
\text { was } 10.9 \%\end{array}$} & $2.5 \pm 0.5$ & $80^{\circ} \mathrm{C}$ & $20 \min$ & $\begin{array}{r}17.75 \% \text { Rdo. } \\
\text { Flour }\end{array}$ \\
\hline 11 & Faba bean hulls (Vicia faba L.) & $\begin{array}{l}\text { Extraction with ethanol, solid to } \\
\text { liquid }(1: 25)\end{array}$ & - & 1.5 & $85^{\circ} \mathrm{C}$ & $80 \min$ & $\begin{array}{r}\text { Rdto. } 12.55 \% \\
13.79 \% \\
\end{array}$ \\
\hline 12 & Durian Rind (Durio zibethinus) & - & - & 2.0 & $90 \circ C$ & $4 \mathrm{~h}$ & - \\
\hline 13 & Lemon peels (Citrus limón) & $\begin{array}{l}\mathrm{HCl}, \mathrm{HNO}_{3} \text {. the best results in } \\
\text { terms of yield } \mathrm{HCL}\end{array}$ & - & $2.5-3.5$ & $70^{\circ} \mathrm{C}$ & $4 \mathrm{~h}$ & $\begin{array}{r}20 \%\left(\mathrm{HNO}_{3}\right) \\
26.3 \\
\% \pm 1.3(\mathrm{HCL}) \\
\end{array}$ \\
\hline \multirow[t]{2}{*}{14} & Flour apple (Malus domestica) & Solute/solvent 1:50 & \begin{tabular}{|l|}
$68.84 \%$ \\
Degree of \\
etherification
\end{tabular} & 2.5 & $97^{\circ} \mathrm{C}$ & $30 \mathrm{~min}$ & $9.73 \%$ \\
\hline & Apple pomace (Malus domestica) & - & - & - & - & - & $6.13 \%$ \\
\hline 15 & $\begin{array}{l}\text { Mango (Mangifera indica) and } \\
\text { ambarella (Spondias cytherea) } \\
\text { peel }\end{array}$ & Hydrochloric acid & - & 1.5 & $85^{\circ} \mathrm{C}$ & $1 \mathrm{~h}$ & $15-20 \%$ \\
\hline 16 & Orange peel & $\begin{array}{l}\text { The best result was obtained with } \\
\text { ammonium oxalate } \\
\text { extraction } 0.25 \%\end{array}$ & - & 3.5 & $75^{\circ} \mathrm{C}$ & $90 \min$ & $30.12 \%$ \\
\hline
\end{tabular}




\begin{tabular}{|c|c|c|c|c|c|c|c|}
\hline \multirow[t]{2}{*}{17} & Apple pomace and citrus peel & Subcritical water & - & - & - & - & $\begin{array}{r}21.95 \% \text { (citrus } \\
\text { peel) }\end{array}$ \\
\hline & & Apple pomace & - & - & - & - & $16.68 \%$ \\
\hline 18 & $\begin{array}{l}\text { Material enriched with beet pulp } \\
\text { (Beta vulgaris) }\end{array}$ & $\begin{array}{l}\text { Ultrasonic-assisted treatment } \\
\text { combined with subcritical water }\end{array}$ & $\begin{array}{l}10.70 \quad \mathrm{MPa} \\
\text { liquid/solid } \\
44.03\end{array}$ & - & $120.72^{\circ} \mathrm{C}$ & $30.49 \mathrm{~min}$ & $24.63 \%$. \\
\hline 19 & Orange juice remainders & $\begin{array}{l}\text { Orange juice waste assisted by } \\
\text { ohmic heating }\end{array}$ & $\begin{array}{l}\text { Voltaje }^{15} \\
\text { V/cm }\end{array}$ & - & $90^{\circ} \mathrm{C}$ & $30 \mathrm{~min}$ & $14.32 \mathrm{~g} / 100 \mathrm{gdm}$ \\
\hline
\end{tabular}

\begin{tabular}{|c|c|c|c|c|c|c|c|}
\hline $\begin{array}{l}\text { Ranking } \\
\text { number }\end{array}$ & Fruit & Method of extraction & Conditions & $\mathrm{pH}$ & Temperature & $\begin{array}{l}\text { Extraction } \\
\text { time }\end{array}$ & $\begin{array}{c}\text { Yield } \\
\text { (Galacturonic } \\
\text { Acid (GalA)) } \\
\end{array}$ \\
\hline & & Conventional method & & & & & $\begin{array}{r}13.53 \mathrm{~g} / 100 \\
\mathrm{gdm}\end{array}$ \\
\hline \multicolumn{8}{|c|}{ Others research fields } \\
\hline 1 & Pomelo peels (Citrus grandis) & $\begin{array}{l}\text { Hot-solvent microwave } \\
\text { extraction (HSME) smaller time } \\
\text { greater yield }\end{array}$ & $520 \mathrm{~W}$ & 2.0 & - & $5.6 \mathrm{~min}$ & $\begin{array}{l}0.05 \text { e } 2.93 \% \\
3.29 \pm 0.15 \%\end{array}$ \\
\hline 2 & Soy hulls (Glycine max) & $\begin{array}{l}\text { Chemical extraction methods } \\
\text { (acid extraction) }\end{array}$ & - & - & - & - & $\begin{array}{r}0.84 \mathrm{~g} \\
\text { Lgalacturonic } \\
\text { acid/g dried } \\
\text { sample }\end{array}$ \\
\hline 3 & $\begin{array}{c}\text { Grapefruit peels (Citrus } \\
\times \text { paradisi) }\end{array}$ & Extraction with acid & - & 1.5 & $120^{\circ} \mathrm{C}$ & - & $(22.55 \%$ \\
\hline 4 & $\begin{array}{l}\text { Palmyra palm } \\
\text { aethiopum Mart) fruit }\end{array}$ & $\mathrm{HNO}_{3} 1 \mathrm{H}$ & - & 2.5 & $30 \mathrm{~min}$ & $80^{\circ} \mathrm{C}$ & $\begin{array}{r}825 \mathrm{~g} / \mathrm{kg}^{1} \\
8.25 \% \\
(+\quad 3.8) \\
\end{array}$ \\
\hline 5 & $\begin{array}{l}\text { Sweet potato residues (Ipomoea } \\
\text { batatas) }\end{array}$ & $\begin{array}{l}\text { Extraction with acid }(\mathrm{HCl}) \\
\text { liquid/solid ratio }(\mathrm{v} / \mathrm{w}) 30: 1 \text {. }\end{array}$ & - & 1.7 & $93^{\circ} \mathrm{C}$ & $2.2 \mathrm{~h}$ & $\begin{array}{r}5.09 \% \text { and } \\
70.03 \%(\mathrm{w} / \mathrm{w})\end{array}$ \\
\hline 6 & $\begin{array}{l}\text { Sweet potato (Ipomoea batatas, } \\
\text { Convolvulaceae) residues }\end{array}$ & $\begin{array}{l}\text { Disodium phosphate } \\
\text { Solution, Liquid/solid ratio } 20: 1\end{array}$ & - & 7.9 & $66^{\circ} \mathrm{C}$ & $3.3 \mathrm{~h}$ & $10.24 \%$ \\
\hline 7 & \multicolumn{2}{|c|}{ Orange shells valencia (Citrus sinensis) } & & 1.5 & $90 \stackrel{\circ}{C}$ & $75 \min$ & \\
\hline
\end{tabular}




\begin{tabular}{|c|c|c|c|c|c|c|c|}
\hline 8 & $\begin{array}{l}\text { Passion Fruit Peels (Passiflora } \\
\text { edulis } f \text {. flavicarpa) }\end{array}$ & $\begin{array}{ll}\begin{array}{l}\text { Conventional } \\
\text { solid/liquid } 1: 25\end{array} & \text { method, } \\
\end{array}$ & - & 2.0 & $70^{\circ} \mathrm{C}$ & $75 \mathrm{~min}$ & $14.6 \%$ \\
\hline 9 & Grapefruit peel (Duncan cultivar) & Extraction with acid & - & 1.5 & $120^{\circ} \mathrm{C}$ & $90 \min$ & $22.55 \%$ \\
\hline \multirow[t]{2}{*}{10} & \multirow[t]{2}{*}{$\begin{array}{l}\text { Jackfruit rinds }(\mathrm{p} / \mathrm{v})(\text { solvent ratio } \\
\text { was adjusted to be } 1: 25(\mathrm{w} / \mathrm{v})\end{array}$} & $\begin{array}{l}\text { Microwave-assisted extraction } \\
\text { (MAE) }\end{array}$ & $450 \mathrm{~W}$ & - & - & - & $16.72-17.63 \%$ \\
\hline & & Extraction with acid & - & - & $90^{\circ} \mathrm{C}$ & $\begin{array}{c}1 \mathrm{~h} \text { and } 10 \\
\min \end{array}$ & $14.59 \%$ \\
\hline 11 & $\begin{array}{l}\text { Saba banana [Musa 'saba' (Musa } \\
\text { acuminata X Musa balbisiana)] } \\
\text { peel wastes }\end{array}$ & Hydrochloric acid $(0.5 \mathrm{~N}) \mathrm{HCl}$ & - & 1.5 & $90^{\circ} \mathrm{C}$ & $4 \mathrm{~h}$ & $17.05 \%$ \\
\hline 12 & Apple juice & Extraction with acid hot $(\mathrm{HCl})$ & - & - & $70^{\circ} \mathrm{C}$ & $1 \mathrm{~h}$ y $30 \mathrm{~min}$ & $52.6 \%$ \\
\hline 13 & $\begin{array}{l}\text { Quince (Cydonia oblonga) } \\
\text { Pomace }\end{array}$ & Extraction with acid $(\mathrm{HCl})$ & - & 2.20 & $80^{\circ} \mathrm{C}$ & $3 \mathrm{~h}$ & $25.2 \mathrm{mg} / \mathrm{g}$ \\
\hline 14 & Passion Fruit Peels (Passiflora & Citric acid & - & 2.0 & - & $75 \min$ & $14.60 \%$ \\
\hline \multirow[t]{2}{*}{$\begin{array}{l}\text { Ranking } \\
\text { number }\end{array}$} & Fruit & Method of extraction & Conditions & $\mathrm{pH}$ & Temperature & $\begin{array}{l}\text { Extraction } \\
\text { time }\end{array}$ & $\begin{array}{c}\text { Yield } \\
\text { (Galacturonic } \\
\text { Acid (GalA)) } \\
\end{array}$ \\
\hline & edulis f. Flavicarpa) & & & & & & \\
\hline 15 & $\begin{array}{l}\text { Jackfruit } \\
\text { heterophyllus) Waste }\end{array}$ & $\begin{array}{l}\text { Sodium } \\
\text { hexametaphosphate/HCl }\end{array}$ & $0.6 \mathrm{~g} / 3 \mathrm{~N}$ & $2.2 \pm 0.02$ & $80 \pm 5^{\circ} \mathrm{C}$ & $15 \mathrm{~min}$ & $15.14 \%$ \\
\hline \multirow[t]{2}{*}{16} & Sugar beet pulp (Beta vulgaris) & \multicolumn{2}{|c|}{$\begin{array}{l}\text { Ultrasound-/microwave-assisted acid. Recovery of } \\
\text { pectin } 636.20 \mathrm{kDa}\end{array}$} & - & - & - & $26.16 \%$ \\
\hline & Uronic acid content & Microwaves. Solid-liquid 1:30 & $295.95 \mathrm{kDa}$ & - & $92^{\circ} \mathrm{C}$ & $37 \min$ & $75.38 \%$ \\
\hline 17 & $\begin{array}{l}\text { Sugar beet pulp (SBP) (Beta } \\
\text { vulgaris) }\end{array}$ & \multicolumn{4}{|c|}{$\begin{array}{l}\text { Compared with gum Arabic, the highest yield SBPP fraction had better } \\
\text { emulsifying activity, but poorer emulsion-stabilizing ability. Using subcritical } \\
\text { water combined with ultrasonic treatment } 42.5 \mathrm{kDa} \text { average molecular weight } \\
\text { (AMW) were obtained from SBP when using distilled water with solid/liquid ratio } \\
\text { of } 1: 81\end{array}$} & $110^{\circ} \mathrm{C}$ & $29.1 \%$ \\
\hline
\end{tabular}

Source: the authors 
Table 3: Number of Ranking (Methodiordinatio), article authors published in the high impact Journals, according to country, number of citations, year of publication and its affiliation to the area of Engineering III (20 articles) and other areas (17)

\begin{tabular}{|c|c|c|c|c|c|c|c|c|}
\hline $\begin{array}{l}\text { Ranking } \\
\text { Number }\end{array}$ & Methodiordinatio & References & Country & Journal name & $\begin{array}{l}\text { Impact factor } \\
2017\end{array}$ & Citations & $\begin{array}{l}\text { Publication } \\
\text { Year }\end{array}$ & Qualis \\
\hline 1 & 100.00 & {$[26]$} & India & $\begin{array}{cc}\text { International journal } & \text { biological } \\
\text { of macromolecules } & \end{array}$ & 3.138 & 20 & 2015 & A2 \\
\hline 2 & Book & [27] & - & - & - & - & - & - \\
\hline 3 & 118.00 & {$[18]$} & China & Food chemistry & 4.052 & 38 & 2015 & $\mathrm{~A} 1$ \\
\hline 4 & 115.00 & [28] & Brazil & Food hydrocolloids & 3.858 & 49 & 2014 & $\mathrm{~A} 1$ \\
\hline 5 & 88.00 & [29] & Spain & $\begin{array}{l}\text { Journal of } \\
\text { Agricultural } \\
\text { Chemistry }\end{array}$ & 2.857 & 21 & 2014 & $\mathrm{~A} 1$ \\
\hline 6 & 133.00 & {$[30]$} & India & Carbohydrate Polymers & 4.219 & 73 & 2013 & $\mathrm{~A} 1$ \\
\hline 7 & 101.00 & [31] & India & $\begin{array}{l}\text { International journal } \\
\text { of macromolecules }\end{array}$ & 3.138 & 21 & 2015 & $\mathrm{~A} 2$ \\
\hline 8 & 90.00 & [32] & Belgium & Journal of Food Science & 1.649 & 90 & 2007 & $\mathrm{~A} 2$ \\
\hline 9 & 83.00 & [33] & Brazil & Journal of Food Engineering & 0.712 & 3 & 2015 & A1 \\
\hline 10 & 89.00 & [34] & Brazil & Journal of Food Science and Technology & 2.203 & 9 & 2015 & B1 \\
\hline 11 & 85.00 & [35] & India & Journal of Food Science and Technology & 2.203 & 5 & 2014 & B1 \\
\hline 12 & 38.00 & {$[36]$} & Malaysia & Journal of Food Science & 1.649 & 18 & 2009 & $\mathrm{~A} 2$ \\
\hline $\begin{array}{l}\text { Ranking } \\
\text { Number }\end{array}$ & Methodiordinatio & References & Country & Journal name & $\begin{array}{l}\text { Impact factor } \\
2017\end{array}$ & Citations & $\begin{array}{l}\text { Publication } \\
\text { Year }\end{array}$ & Qualis \\
\hline 13 & 37.00 & [37] & Italy & $\begin{array}{l}\text { International Journal of Food Science \& } \\
\text { Technology }\end{array}$ & 1.384 & 17 & 2009 & B1 \\
\hline 14 & 146.00 & [38] & Brazil & $\begin{array}{c}\text { Brazilian Archives of } \\
\text { Biology } \quad \text { and Technology }\end{array}$ & 0.468 & 166 & 2005 & B2 \\
\hline 15 & 34.00 & [39] & France & $\begin{array}{l}\text { International Journal of Food Science \& } \\
\text { Technology }\end{array}$ & 1.384 & 14 & 2009 & B1 \\
\hline 16 & 26.00 & [25] & Turkey & Carbohydrate polymers & 4.219 & 114 & 1999 & A1 \\
\hline
\end{tabular}


Revista ECIPerú

$17 \quad 153.00$

$18 \quad 105.00$

$19 \quad 100.00$

93.00

[42]

88.00

93.00

80.00

65.00

58.00

92.00

95.00

72.00

9

$10 \quad 81.00$

$11 \quad 85.00$

$12 \quad 100.00$

$13 \quad 72.00$

14

15

16

95.0076 .00

84.00

81.00

[20]

[40]

[41]

[44]

[46]

[48]

[54]

17

[55]
Volumen 15, número 1

China Food hydrocolloids

Food chemistry

4.090

China

Iran

Chemical engineering and processing

4.052

2.154

\section{Other area of research}

China

USA

EE.UU

EE.UU

China

China

LWT.Food Science and Technology $\quad 2.416$

LWT.Food Science and Technology 2.416

LWT.Food Science and Technology $\quad 2.416$

LWT.Food Science and Technology

2.416

International Journal of Food Science and 1.504

\section{Technology}

International Journal of Food Science \& $\quad 1.504$

Technology

Brazil

Malaysia

Pakistan

Malaysia

Malaysia

Malaysia

Argentina

Malaysia

Bangladesh

China

China
2.571

Agriculture and Agricultural

$$
\text { Science Procedia }
$$

Current Nutrition and Food Science

International Food Research Journal

International Food Research Journal

Food Science and Technology International

Agriculture and Agricultural Science Procedia International Journal of Food Science and

Technology

International Journal of Food Science and

Technology
Food and Bioprocess Technology

$\begin{array}{lll}83 & 2014 & \text { A1 } \\ 29 & 2015 & \text { A1 } \\ - & 2017 & \text { A2 }\end{array}$

A1

A1

A2

A1

A1

A1

A1

C

C

A1

C

B1

B1

B1

B1

B1

C

C

C 
Table 4: Number of articles according to impact factor Journals selected

\begin{tabular}{|c|c|c|}
\hline Journal identification (Engineering III) & Journal Amount & Qualis \\
\hline $\begin{array}{l}\text { International journal of biological } \\
\text { macromolecules }\end{array}$ & 2 & A2 \\
\hline Food chemistry & 2 & A1 \\
\hline Food hydrocolloids & 2 & A1 \\
\hline $\begin{array}{l}\text { Journal of Agricultural and Food } \\
\text { Chemistry }\end{array}$ & 1 & $\mathrm{~A} 1$ \\
\hline Carbohydrate Polymers & 2 & A1 \\
\hline Journal of Food Science & 2 & $\mathrm{~A} 2$ \\
\hline Journal of Food Engineering & 1 & A1 \\
\hline Journal of Food Science and Technology & 2 & B1 \\
\hline $\begin{array}{l}\text { International Journal of Food Science \& } \\
\text { Technology }\end{array}$ & 3 & B1 \\
\hline $\begin{array}{l}\text { Brazilian Archives of Biology and } \\
\text { Technology }\end{array}$ & 1 & B2 \\
\hline Chemical engineering and processing & 1 & A2 \\
\hline Total & 19 & \\
\hline \multicolumn{3}{|l|}{ Outside Engineering III } \\
\hline LWT - Food Science and Technology & 4 & A1 \\
\hline $\begin{array}{l}\text { International Journal of Food Science and } \\
\text { Technology }\end{array}$ & 4 & C \\
\hline Food and Bioprocess Technology & 1 & A1 \\
\hline Current Nutrition and Food Science & 1 & B1 \\
\hline International Food Research Journal & 3 & B1 \\
\hline $\begin{array}{l}\text { Food Science and Technology } \\
\text { International }\end{array}$ & 1 & B1 \\
\hline $\begin{array}{l}\text { Agriculture and Agricultural Science } \\
\text { Procedia }\end{array}$ & 3 & C \\
\hline Total & 17 & \\
\hline
\end{tabular}

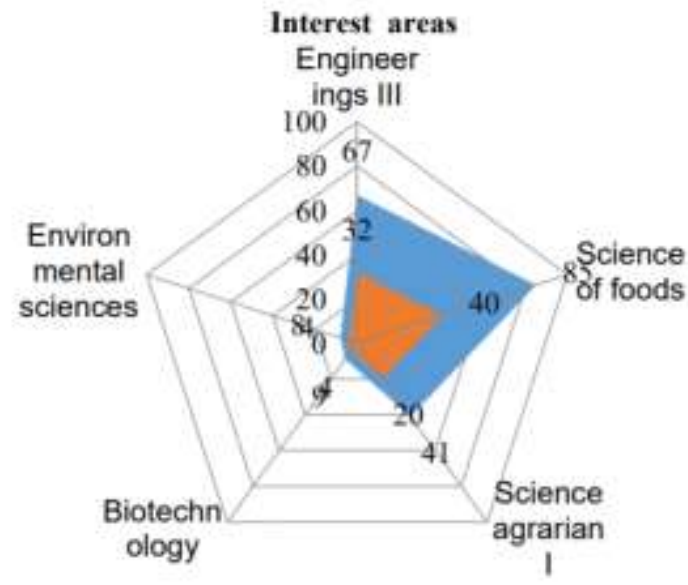

Source: the authors

Figure 1: Amount of revision. 


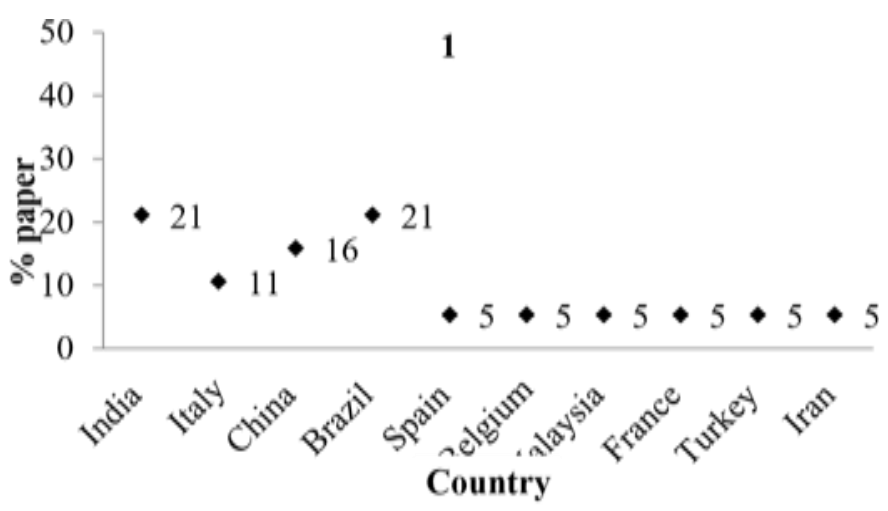

Figure 2: Percentage of Journals found by research country. Source: the authors

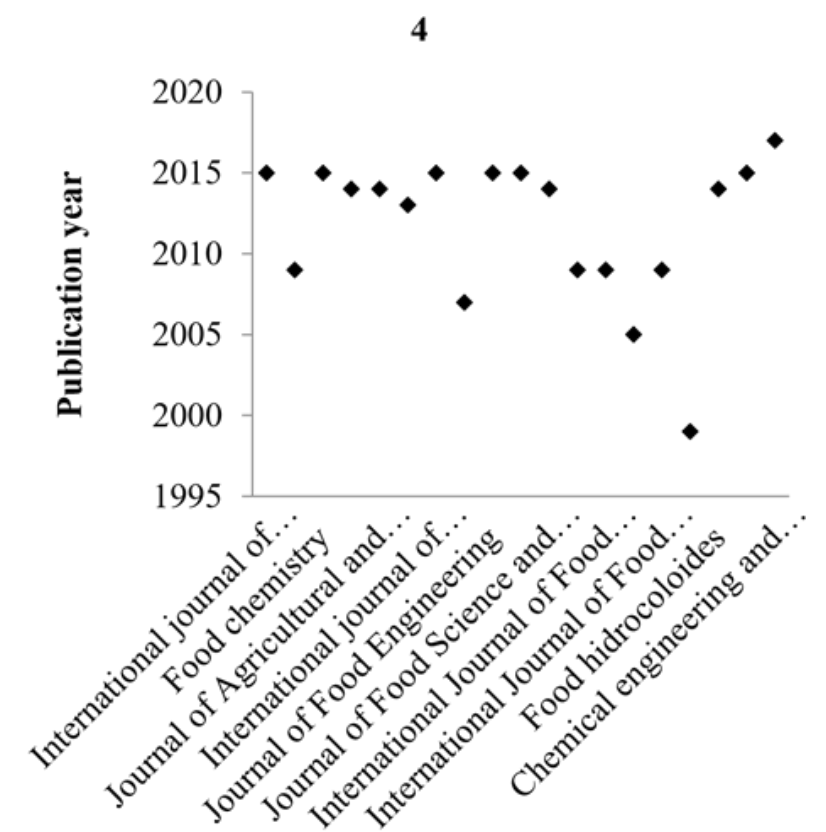

Yournal Engineering III

Figure 3: Journals found by year of publication. Source: the authors

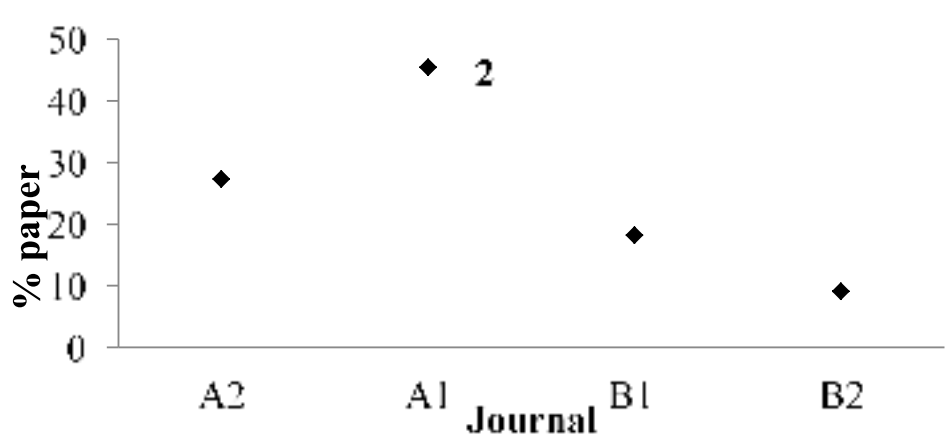

Figure 4: Percentage of Journals by qualis pertaining to Engineering III. Source: the authors

According to Figure 2 and 4, we can deduce that the Journals were included from year 1995 to year 2017. Of them $16 \%$ belong to International
Journal of Food Science \& Technology. $11 \%$ for each one of the following Journals: International Journal of Biological Macromolecules, Food 
chemistry, Food hydrocolloids, Carbohydrate polymers, Journal of food, Journal of Food Science and Technology; in the same way for each one $5 \%$ belong a Journal of agricultural and food chemistry, Journal of food engineering, Brazilian archives of biology and technology and Chemical engineering and processing (Figure 4).

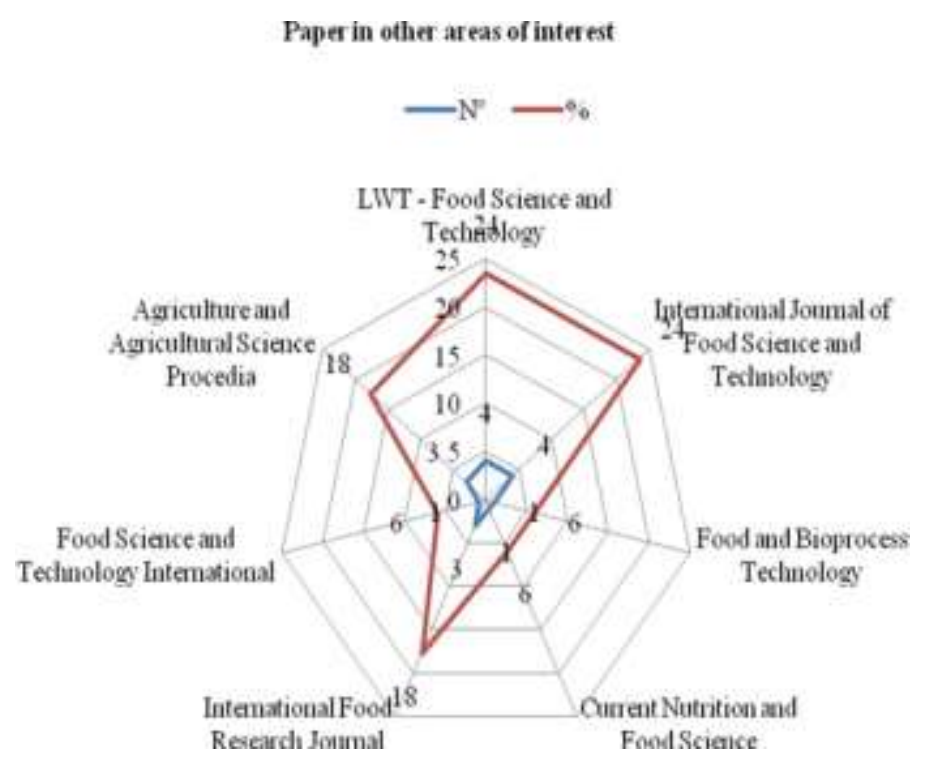

Figure 5: Journals found in other research areas. Source: the authors

Regarding to publications in other areas, $24 \%$ belongs to the Food Science and technology, Journals of high impact A1 y $24 \%$ in the International Journal of Food Science and Technology with impact factor $\mathrm{C}$. The smaller number of found articles was in the Journals such as Agriculture and Agricultural Science Procedia, International Food Research Journal, Food and Bioprocess Technology, Current Nutrition and Food Science, Food Science and Technology International respectively as one is in the Figure (3, 4) and Figure 5.

\section{Discussion}

The key to the knowledge transfer process is the assimilation of the results and the effects of the application [1] ensures that knowledge can share others that do not have "explicit (objective) and Tacitus (subjective) knowledge" [56].

In this way, knowledge transfer takes place; in addition, it is possible to distinguish by this method, the searches of traditional information, taking place an innovation when using the reference, with technological information software.

This paper demonstrates the search methodology with, case study of hydrocolloids (pectin). Waiting that it could be used as guide for others research as a method to search for information. The "quails" as shown by the results tables are the quality classification and the impact factor of the Magazines; regarding the area of Engineering III in Brazil.

The method "Methodiordinatio" is an extraordinary methodology because it classifies the Journals according to their impact factor, year, number of citations and quails. This method saves time in making decision at the time of choosing the papers. On the other hand, bibliographic references were used, such as Zotero, EndNote, and JabRef, which helps to organize articles in the simplest way. In this case study, diverse theories were found to discuss. During the life cycles of fruit, great coproducts are generated from agricultural processing. For example, in Brazil, 183.5 thousand tons' metric of solid wastes are generated [57]. In the processing of fruits, a great amount of byproducts, especially seeds and rinds, are discarded in the environment causing organic contamination [24, 58]. An alternative to avoid this problem is to obtain pectin, but we can recognize that seeds have attributed beneficial properties to their possible content in compounds with high added value, especially bioactive isothiocyanates as well as phenolic compounds, that can be used for 
application in nutraceutical supplements, dietetic additives. It is possible to find new foods (texturizing additives) and pharmaceutical products in the food industry $[58,59]$.

In this case study, papaya peel with microwaves extraction for 140 seconds obtained a yield of 25.41 $\%$ pectin [31]. In the same way, lemon rinds as a raw material in the extraction yield of $\mathrm{HCL}$ of $26.3 \pm 1.3$ [37].

The classic treatments (grinding, heating) and the different alternative treatments until now used in the industry to proceed easier extractions degrade and alter the structure of the pulp (cellular membranes and walls) uncontrollable, unfortunately, the flesh completely disorganized pulp loses its selectivity and becomes permeable not only for cellular compounds, but also for undesirable compounds (impurities) presents in the extract [58].

Another new promising and effective technology for the pectin extraction is by means of the ultrasound method, using subcritical water have been performance values of yield $26.16 \%[54,60]$. However, pectins are rich in galacturonic acid [61], FAO and UE stipulate that pectins must consist of at least $65 \%$ galacturonic acid in a dry and ash free of ashes.

Recovery and valuation of the crops, using technologies that include the reduction of losses to improve food security, maintain sustainability in agricultural practice with minimal environmental impact [45].

\section{Conclusions}

Since it has been described previously, a case study was carried out in the area of hydrocolloids with pectin using key words: "Anthropotechnology, Knowledge management and innovation, technology transfer, pectin. Search was made in the data bases Scopus, CienceDirect y Web of Science, with the methodology "Methodiordinatio".

\section{Limitations of the investigation}

With respect to the amplitude of consultations is it data bases.

\section{Acknowledgements}

The authors are grateful to the UTFPR. Santos Pedraza Guevara, her Doctoral scholarship fellow by
Organization of American States OEA - GCUB in Engineering of the Production, to the Research Group of Management of Agro-industrial Knowledge from UTFPR.

\section{References}

[1] M. Gilbert and M. CordeyHayes, Technovation, 16 (1996) 301-312.

[2] M. H. Canteri-Schemin, H. C. R. Fertonani, N. Waszczynskyj and G. Wosiacki, Brazilian Archives of Biology and Technology, 48 (2005) 259-266.

[3] J.-A. Johannessen, J. Olaisen and B. Olsen, International journal of information management, 21 (2001) 3-20.

[4] M. J. Donate and J. D. S. de Pablo, Journal of Business Research, 68 (2015) 360-370.

[5] R. N. Pagani, J. L. Kovaleski and L. M. Resende, Scientometrics, 105 (2015) 21092135.

[6] N. Shibata, Y. Kajikawa, Y. Takeda and K. Matsushima, Technovation, 28 (2008) 758775.

[7] D. J. de Solla Price, Technology and Culture, 1965, 553-568.

[8] N. d. Santos, A. d. A. DUTRA, F. Fialho, R. Proença and C. Righi, Curitiba: Gênesis, 1997, 49-51.

[9] A. Wisner, Quand voyagent les usines: essai d'anthropotechnologie, Syros, 1985.

[10] R.-S. Chen and C.-H. Hsiang, Information Sciences, 177 (2007) 570-586.

[11] H. M. Cooper, Synthesizing research: A guide for literature reviews, Sage, 1998.

[12] A. Reisman, Omega, 33 (2005) 189-202.

[13] A. Khan, M. Butt, M. Randhawa, R. Karim, M. Sultan and W. Ahmed, International Food Research Journal, 21 (2014).

[14] S.-M. Tseng, Expert systems with applications, 35 (2008) 150-160.

[15] H.-W. Kim, Y. J. Lee and Y. H. B. Kim, LWTFood Science and Technology, 64 (2015) 1071-1077.

[16] M. H. Canteri, L. Moreno, G. Wosiacki and A. d. P. Scheer, Polímeros, 22 (2012) 149157.

[17] W. Wang, X. Ma, Y. Xu, Y. Cao, Z. Jiang, T. Ding, $X$. Ye and D. Liu, Food chemistry, 178 (2015) 106-114.

[18] B. B. Koubala, G. Kansci, C. Garnier, I. L. Mbome, S. Durand, J. F. Thibault and M. C. Ralet, International journal of food science \& technology, 44 (2009) 1809-1817. 
[19] X. Wang, Q. Chen and X. Lü, Food Hydrocolloids, 38 (2014) 129-137.

[20] Z. Yan Yan, M. Tai Hua and Z. Miao, International Journal of Food Science \& Technology, 48 (2013) 778-785.

[21] Q. Chen, Z. Hu, F. Y.-D. Yao and H. Liang, LWT-Food Science and Technology, 66 (2016), 538-545.

[22] L. R. Adetunji, A. Adekunle, V. Orsat and V. Raghavan, Food Hydrocolloids, 62 (2017) 239-250.

[23] B. B. Koubala, S. Christiaens, G. Kansci, A. M. Van Loey and M. E. Hendrickx, Food research international, 55 (2014) 215-221.

[24] F. Kar and N. Arslan, Carbohydrate Polymers, 40 (1999) 277-284.

[25] J. P. Maran, International journal of biological macromolecules, 73 (2015) 9298.

[26] D. S. Robinson, Bioquímica y valor nutritivo de los alimentos, Editorial Acribia, SA, 1991.

[27] F. L. Seixas, D. L. Fukuda, F. R. Turbiani, P. S. Garcia, L. d. O. Carmen, S. Jagadevan and M. L. Gimenes, Food Hydrocolloids, 38 (2014) 186-192.

[28] B. n. Gómez, B. Gullón, C. Remoroza, H. A. Schols, J. C. Parajó and J. L. Alonso, Journal of agricultural and food chemistry, 62 (2014) 9769-9782.

[29] J. P. Maran, V.

Sivakumar,

$\mathrm{K}$.

Thirugnanasambandham and R. Sridhar, Carbohydrate polymers, 97 (2013) 703709.

[30] J. P. Maran and K. A. Prakash, International Journal of Biological Macromolecules, 2015, 73, 202-206.

[31] H. Garna, N. Mabon, C. Robert, C. Cornet, K. Nott, H. Legros, B. Wathelet and M. Paquot, J Food Sci, 72 (2007) C001-009.

[32] K. Zanella and O. P. Taranto, Journal of Food Engineering, 166 (2015) 111-118.

[33] S. S. Venzon, M. H. G. Canteri, D. Granato, B. D. Junior, G. M. Maciel, A. P. Stafussa and C. W. I. Haminiuk, Journal of Food Science and Technology, 52 (2015) 41024112.

[34] J. P. Maran, International journal of biological macromolecules, 73 (2015) 9298.

[35] J. P. Maran, V. Sivakumar, K. Thirugnanasambandham and R. Sridhar, Carbohydrate polymers, 97 (2013) 703709.

[36] J. P. Maran and K. A. Prakash, International Journal of Biological Macromolecules, 2015, 73, 202-206.
[37] H. Garna, N. Mabon, C. Robert, C. Cornet, K. Nott, H. Legros, B. Wathelet and M. Paquot, J Food Sci, 72 (2007) C001-009.

[38] K. Zanella and O. P. Taranto, Journal of Food Engineering, 166 (2015) 111-118.

[39] S. S. Venzon, M. H. G. Canteri, D. Granato, B. D. Junior, G. M. Maciel, A. P. Stafussa and C. W. I. Haminiuk, Journal of Food Science and Technology, 52 (2015) 41024112.

[40] M. Korish, Journal of Food Science and Technology, 52 (2015) 6061-6066.

[41] W. W. Weng, A. A. F.M. and E. A. Mat, Journal of Food Science, 74 (2009) C637C641.

[42] S. Maurizia, P. Monica, P. Maurizio, G. Samuele and F. Cristian, International Journal of Food Science \& Technology, 44 (2009) 574-580.

[43] M. H. Canteri-Schemin, H. C. R. Fertonani, N. Waszczynskyj and G. Wosiacki, Brazilian Archives of Biology and Technology, 48 (2005) 259-266.

[44] K. B. Bargui, K. Germain, G. Catherine, M. I. Lape, D. Sylvie, T. Jean François and R. Marie Christine, International Journal of Food Science \& Technology, 44 (2009) 1809-1817.

[45] H.-m. Chen, X. Fu and Z.-g. Luo, Food Chemistry, 168 (2015) 302-310.

[46] H. Saberian, Z. Hamidi-Esfahani, H. Ahmadi Gavlighi and M. Barzegar, Chemical Engineering and Processing: Process Intensification, 117 (2017) 154161.

[47] Q. Chen, Z. Hu, F. Y.-D. Yao and H. Liang, LWT - Food Science and Technology, 66 (2016) 538-545.

[48] H.-W. Kim, Y. J. Lee and Y. H. B. Kim, LWT - Food Science and Technology, 64 (2015) 1071-1077.

[49] W. Wang, X. Ma, P. Jiang, L. Hu, Z. Zhi, J. Chen, T. Ding, X. Ye and D. Liu, Food Hydrocolloids, 61 (2016) 730-739.

[50] S. Assoi, K. Konan, L. T. Walker, R. Holser, G. N. Agbo, H. Dodo and L. Wicker, LWT Food Science and Technology, 58 (2014) 214-221.

[51] Z. Chunpeng and M. Taihua, International Journal of Food Science \& Technology, 46 (2011) 2274-2280.

[52] C. F. de Oliveira, P. D. Gurak, F. CladeraOlivera, L. D. F. Marczak and M. Karwe, Food and Bioprocess Technology, 9 (2016) 1021-1030. 
[53] S. Q. Liew, N. L. Chin and Y. A. Yusof, Agriculture and Agricultural Science Procedia, 2 (2014) 231-236.

[54] P. C. Koh, C. M. Leong and M. A. Noranizan, International Food Research Journal, 21 (2014) 2091-2097.

[55] K. A. T. Castillo-Israel, S. F. Baguio, M. D. B. Diasanta, R. C. M. Lizardo, E. I. Dizon and M. I. F. Mejico, International Food Research Journal, 22 (2015) 202-207.

[56] K. Gazala, F. A. Masoodi, H. D. Masarat, B. Rayees and M. W. Shoib, International Food Research Journal, 24 (2017) 594599.

[57] V. A. Brown, J. E. Lozano and D. B. Genovese, Food Science and Technology International, 20 (2013) 83-98.

[58] R. Begum, M. G. Aziz, M. B. Uddin and Y. A. Yusof, Agriculture and Agricultural Science Procedia, 2 (2014) 244-251.

[59] P. Xiao Yan, M. Tai Hua, Z. Miao, S. Hong Nan, C. Jing Wang and Y. Ming, International Journal of Food Science \& Technology, 50 (2015) 758-765.
[60] C. Hai Ming, F. Xiong, A. A. M. and L. Zhi Gang, International Journal of Food Science \& Technology, 50 (2015) 13241330.

[61] A. Brennan and L. Dooley, Technovation, 25 (2005) 1388-1399.

[62] A. Antunes da Luz, A. C. de Francisco, S. F. Miranda Santos, A. Mesquita Soares and J. L. Kovaleski, Interciencia, 41 (2016).

[63] O. Parniakov, E. Roselló-Soto, F. J. Barba, N. Grimi, N. Lebovka and E. Vorobiev, Food Research International, 77 (2015) 711-717.

[64] J. F. Ayala-Zavala, V. Vega-Vega, C. RosasDomínguez, H. Palafox-Carlos, J. A. VillaRodriguez, M. W. Siddiqui, J. E. Dávila-Aviña and G. A. González-Aguilar, Food Research International, 44 (2011) 1866-1874.

[65] L. Chen, J. Liu, Y. Zhang, B. Dai, Y. An and L. Yu, Journal of Agricultural and Food Chemistry, 2015, 63, 3219-3228.

[66] D. Karnik, J. Jung, S. Hawking and L. Wicker, Food Hydrocolloids, 60 (2016) 179185. 